\title{
Low temperature sputter-deposited ZnO films with enhanced Hall mobility using excimer laser post-processing
}

\section{Tsakonas ${ }^{a, e}$, V.L. Kuznetsov ${ }^{b}$, W.M. Cranton ${ }^{c}$, N. Kalfagiannis ${ }^{a}$, K.M. Abusabee ${ }^{d}$, D.C. Koutsogeorgis ${ }^{a}$, N. Abeywickrama ${ }^{a}$, and P.P. Edwards ${ }^{b}$}

${ }^{a}$ School of Science and Technology, Nottingham Trent University, Nottingham, NG11 8NS, UK.

${ }^{b}$ Department of Chemistry, Inorganic Chemistry Laboratory, University of Oxford, South Parks Rd, OX1 3QR, UK.

${ }^{c}$ Faculty of Arts, Computing, Engineering and Sciences, Sheffield Hallam University, Howard St., Sheffield, S1 1WB, UK.

${ }^{\mathrm{d}}$ Electrical and Computer Engineering Department, Faculty of Engineering, Al-Mergib University, Al khums , Box 40161, Libya.

e e-mail: costas.tsakonas@ntu.ac.uk

\begin{abstract}
We report the low temperature $\left(\mathrm{T}<70^{\circ} \mathrm{C}\right)$ fabrication of $\mathrm{ZnO}$ thin films $(\sim 140 \mathrm{~nm})$ with Hall mobility of up to $17.3 \mathrm{~cm}^{2} \mathrm{~V}^{-1} \mathrm{~s}^{-1}$ making them suitable for thin film transistor (TFT) applications. The films were deposited by rf magnetron sputtering at $\mathrm{T}<70{ }^{\circ} \mathrm{C}$ and subsequently laser processed in ambient temperature in order to modify the Hall mobility and carrier concentration. Medium-to-low energy laser radiation densities and a high number of pulses were used to avoid damaging the films. Laser annealing of the films after aging in the lab under $25 \%-35 \%$ relative humidity and at an average illuminance of 120 lux resulted in an overall higher mobility and relatively low carrier concentration in comparison to the non-aged films that were laser processed immediately after deposition. A maximum overall measured Hall mobility of $17.3 \mathrm{~cm}^{2} \mathrm{~V}^{-1} \mathrm{~s}^{-1}$ at a carrier density of $2.3 \times 10^{18} \mathrm{~cm}^{-3}$ was measured from a $1 \mathrm{G} \Omega$ as deposited and aged film after the laser treatment. We suggest that the aging of non-processed films reduces structural defects mainly at grain boundaries by air species chemisorption, with concomitant increase in thermal conductivity so that laser processing can have an enhancing effect. Such a processing combination can act synergistically and produce suitable active layers for TFT applications with low temperature processing requirements.
\end{abstract}


Keywords: excimer laser annealing, rf magnetron sputtering, grain boundary scattering, $\mathrm{ZnO}$. 


\section{Introduction}

Zinc oxide $(\mathrm{ZnO})$ has been proclaimed as the silicon of the $21^{\text {th }}$ century ${ }^{1}$ because it is a non-toxic, low cost and technologically important material which is used in many applications such as transparent conducting films, sensors, Surface Acoustic Devices (SAW), thin film transistors, photodiodes and UV light emitting diodes ${ }^{2}$. It has attracted considerable attention recently due to its high transparency in the visible region and its electrical properties that can be tailored, by varying mobility $(\mu)$ and carrier density (n), according to the requirements of the application using either intrinsic or doped material. The intrinsic material is mostly used in thin film transistors (TFTs) where a high $\mu$ and low $n$ are required. Whereas doped $\mathrm{ZnO}$ is preferred for low emissivity glass coatings that require high enough $\mathrm{n}$, to achieve the required infrared reflectivity. $\mathrm{ZnO}$ can be easily deposited as a thin film at room temperature on glass and plastic substrates using deposition methods suitable for reel to reel manufacture.

The electrical characteristics of $\mathrm{ZnO}$ thin films as determined by the mobility and carrier concentration are highly sensitive to atomic defects, microstructure and impurities, with annealing processes typically used to ensure good electrical performance and stability. As an alternative to high temperature processing, photonic processing techniques are of interest, combined with low temperature deposition to facilitate the use of plastic and flexible substrates.

Photonic processing, using techniques such as excimer laser annealing (ELA), is of interest for low temperature post deposition processing since the high absorption coefficient at UV and near-UV wavelengths facilitates localised energy dissipation within tens of nanometres from the surface, hence providing the opportunity to process a channel layer with minimal effect on the underlying substrates $^{3,4}$ or multilayer structures with heat sensitive components ${ }^{3,5}$.

The applications of intrinsic $\mathrm{ZnO}$ are mostly limited to TFT and sensor devices. However, these two applications have diverse requirements as to the desired crystal structure for the optimisation of these devices since a TFT requires a crystal structure with as little defects and grain boundaries as possible while a porous structure is desired for sensors. The dependence of the conduction process on grain 
boundaries in intrinsic $\mathrm{ZnO}$ complicates the processing requirements. The majority of recent studies that investigated the effect of photonic processing on the electrical properties involved extrinsic $\mathrm{ZnO}$ thin films. Some studies also reported on the effect of laser processing on intrinsic ZnO TFTs. These studies using various laser systems such as $\mathrm{XeCl}^{3}, \mathrm{KrF}^{6,7}, \mathrm{Nd}: \mathrm{YAG}^{4,8,9}$, indicated that laser annealing has the potential to induce enhancements in crystallinity and field effect mobility which are comparable to the effects produced by thermal annealing at high temperatures. From the previous studies it is evident that excimer laser annealing can be employed alongside sputtering to produce commercially viable $\mathrm{ZnO}$ based TFTs with minimal production steps ${ }^{3}$.

Recently it was demonstrated that excimer laser annealing of stoichiometric $\mathrm{ZnO}$ thin films deposited on silicon by High Target Utilisation Sputtering (HiTUS) produced strong excitonic emission with minimal defect emission ${ }^{10}$ when compared to other annealing methods like rapid thermal annealing and furnace annealing. Furthermore, deposition by sputtering is suitable for scale up applications and consequently is of interest for TFT fabrication using transparent and flexible substrates, where the potential for large area, low temperature deposition is desired. Hence, it is of particular interest to investigate and analyse the electrical properties modification of $\mathrm{ZnO}$ films deposited by sputtering followed by laser annealing.

There have been a lot of publications trying to evaluate and alter the properties of grain boundaries since they control the electron transport in polycrystalline films both during and after deposition. This article presents electrical and structural evidence that environmental aging with laser annealing can alter the electrical properties of very resistive films $(R>1 G \Omega)$, through grain boundary defect state modification. The current report aims to be qualitative than quantitative with the intention to unravel the underlying mechanism for such a transformation in electrical properties so that a shorter processing duration might be effected. Specifically, we report on $248 \mathrm{~nm}$ excimer laser processing of $\mathrm{ZnO}$ (deposited at low temperatures $\left(\mathrm{T}<70{ }^{\circ} \mathrm{C}\right.$ ) by $\mathrm{RF}$ magnetron sputtering) and establish relations between the laser processing parameters and the physical changes induced in the thin films such as the crystal structure, grain size, Hall mobility and carrier concentration. We applied multi-pulse irradiation at relatively low fluence $\left(50-83 \mathrm{~mJ} \mathrm{~cm}^{-2}\right)$ on $140 \mathrm{~nm}$ thick films, in contrast to ref 2 that 
used very high fluence $\left(350 \mathrm{~mJ} \mathrm{~cm}^{-2}\right)$ and a single pulse to melt the $20 \mathrm{~nm}$ thick $\mathrm{ZnO}$ layer. We demonstrate that the Hall mobility is almost constant in the temperature range $78 \mathrm{~K}-360 \mathrm{~K}$ for the sample with the largest achievable Hall mobility $\left(17.3 \mathrm{~cm}^{2} \mathrm{~V}^{-1} \mathrm{~s}^{-1}\right)$ at quite low carrier concentration $\left(2.3 \times 10^{18} \mathrm{~cm}^{-3}\right)$, suitable for TFT applications.

The exact nature of grain boundary modification is difficult to assess unequivocally since grain boundaries represent a small fraction of a thin film and direct probing methods like EELS (Electron Energy Loss Spectroscopy) are limited by spatial resolution. Environmental gasses (carbon dioxide, nitrogen or oxygen) in the presence of small quantities of water vapour and aided by low levels of white light seem to create the necessary conditions to bring about suitable modification of the chemical states at the grain boundaries. Any such process is probably also aided by random atom movement at room temperature. In the current investigation long environmental aging was required since the films were highly resistive and quite thick but under amenable conditions of suitable starting material shorter periods might be enough. Furthermore the physical processes discussed therein can have relevance to thin film science.

\section{Experimental details}

$\mathrm{ZnO}$ thin films were deposited by $\mathrm{RF}$ magnetron sputtering from a $\mathrm{ZnO}$ ceramic source target (purity of 99.99\%); no intentional substrate heating was applied, with the ultimate substrate temperatures attained being $<70^{\circ} \mathrm{C}$.

The $\mathrm{ZnO}$ films were grown on $7.5 \mathrm{~cm} \times 5 \mathrm{~cm}$ soda lime glass substrates. The glass substrates were cleaned with acetone followed by isopropanol and baked at $270^{\circ} \mathrm{C}$ in vacuum for $30 \mathrm{~min}$ before any deposition took place. Depositions were carried out at least 2 hours after the substrate baking when the temperature of the substrate had cooled to ambient temperature. The sputtering system had a base pressure of $1.2 \times 10^{-7}$ Torr, the distance between the substrate and the source ( 3 inch diameter $\mathrm{ZnO}$ target) was $10 \mathrm{~cm}$. The total sputtering pressure was maintained at 2 mTorr during deposition. The substrate holder rotated at $12 \mathrm{rpm}$ and the target was positioned at $130^{\circ}$ to the substrate holder so that there is minimal thickness variation across the glass substrates. The different films were produced by 
varying the rf power and the oxygen/argon ratio by controlling the flow rate of two separate gas lines connected to two cylinders containing $100 \%$ argon and $2 \%$ oxygen/argon mixture respectively. The flow rate of the two gas lines was regulated so as the oxygen flow rate comprised a certain percentage $(0.2 \%-2 \%)$ of the total argon flow rate. The total flow rate, for the combined gas lines, feeding the deposition chamber, was between 9 and $13 \mathrm{sccm}$ depending on the required oxygen percentage. Films deposited at oxygen/argon flow rate percentage lower than $0.2 \%$ were mostly metallic and semi-transparent. The rf power was varied from $40 \mathrm{~W}$ to $300 \mathrm{~W}$ independently from the variation of oxygen content in the sputtering gas. Hence for a systematic study, the various samples are identified by the deposition condition (comprising of the rf power and oxygen/argon flow rate) and a serial number. The thickness of the films was $140 \pm 10 \mathrm{~nm}$ defined by in-situ and real-time interferometry measurement. A number of films with high as-deposited resistivity, outside the measuring range of the Hall system, were stored in closed petri-dishes in the laboratory $\left(20^{\circ} \mathrm{C}-28^{\circ} \mathrm{C}\right.$ with $25 \%-35 \%$ relative humidity) and aged for 20 - 25 months. The films were exposed to fluorescent light during this period at an average illuminance of 120 lux. Laser processing was undertaken in air using a KrF Lambda Physik 305i excimer laser (248 nm) and a beam delivery system providing a homogenised $13 \mathrm{~mm} \times 13 \mathrm{~mm}$ square beam at the sample plane (hence covering the full area of the $1 \mathrm{~cm} \times 1 \mathrm{~cm}$ Hall samples). This configuration was also used previously for laser processing of thin phosphor films for foldable displays ${ }^{11}$ and has been demonstrated to facilitate large area processing of display substrates via a sample step and repeat process. A range of processing parameters was used by varying the fluence $\left(48-295 \mathrm{~mJ} \mathrm{~cm}^{-2}\right)$ and the number of pulses (1-6000) at a pulse frequency of $10 \mathrm{~Hz}$.

The samples were diced to $1 \mathrm{~cm} \times 1 \mathrm{~cm}$ size areas, as required for Hall effect measurements, and subsequently laser processed. All irradiated $1 \mathrm{~cm} \times 1 \mathrm{~cm}$ size samples, for a certain deposition condition, were sourced from films produced in a single deposition run. Hall effect measurements over the $78 \mathrm{~K}$ to $360 \mathrm{~K}$ range were performed by the Van der Pauw technique using an Ecopia HMS 3000 station with a $0.55 \mathrm{~T}$ magnetic field and a cryogenic cooling system using indium contacts. The final mobility and carrier concentration values were averaged over 3-6 measurements. The sheet 
resistance of the samples was measured using a four point probe technique. The thickness was confirmed using a stylus profilometer (Dektak).

Cross-sectional Transmission Electron Microscopy (TEM) was performed for selected films using a JEOL2000fx operated at $200 \mathrm{keV}$. High Resolution Transmission Electron Microscopy was performed using a FEI Titan Themis 300 fitted with Super-X EDX, Gatan OneView camera and a Quantum ER imaging filter at $300 \mathrm{kV}$. The cross-sectional cuts were removed from the centre of each sample following the established trench technique ${ }^{12}$. The morphological features of the $\mathrm{ZnO}$ thin films were obtained by Atomic Force Microscopy (AFM) performed with a Dimension Icon Scanning Probe Microscope (Bruker). The AFM system was mounted in an anti-vibrational air stable granite stage installed inside an acoustic enclosure using a standard tapping mode for better image acquisition. XPS depth profile analysis was carried out at the National EPSRC XPS User's service (NEXUS) at Newcastle University (UK) using a Thermo Scientific (UK) K-Alpha instrument with an Al $\mathrm{K}_{\alpha} \mathrm{X}$-ray source $(1486.7 \mathrm{eV})$. Nano-indentation measurements were carried out with a Mitutoyo MVK-H1 instrument and a Vickers type pyramidal diamond square indenter with an angle of 136 degrees between opposite faces using a 25 gr load. Transmittance data were collected using a Filmetrics F20 thin film analyser in the range $400 \mathrm{~nm}$ to $1000 \mathrm{~nm}$ and, a Cary 5000 UV-visible-nearIR spectrophotometer in the range 200 to $2500 \mathrm{~nm}$. After deposition the transmittance and reflectance spectra at various areas across the whole substrate area for selected films were investigated and showed small disparity. The absorption coefficient of selected films was obtained using the Scout optical package (http://www.wtheiss.com) to analyse the transmittance and reflectance spectra collected using the Filmetrics system.

\section{Results}

\subsection{Room temperature Hall effect measurements - As-deposited (non-aged) samples}

Most as-deposited films had an electrical resistance of more than $1 \mathrm{G} \Omega$ and could not be measured by the four point probe technique. Significant resistivity decrease using laser processing was only possible for three films that possessed measurable finite sheet resistance before any laser processing 
took place. Two of these films were deposited using $200 \mathrm{~W}$ and $300 \mathrm{~W}$ rf power at $0.2 \%$ and $0.5 \%$ oxygen/argon flow rate respectively. A smaller resistivity decrease was also observed for the third film deposited using $300 \mathrm{~W}$ and $0.2 \%$ oxygen gas content but the achieved Hall mobility following laser processing was small.

For the results presented below the following notation for the range of investigated films is used: (i) $300 \mathrm{~W}$ at $0.2 \% \mathrm{O}_{2} / \mathrm{Ar}$, (ii) $300 \mathrm{~W}$ at $0.5 \% \mathrm{O}_{2} / \mathrm{Ar}$, (iii) $300 \mathrm{~W}$ at $0.7 \% \mathrm{O}_{2} / \mathrm{Ar}$, (iv) $300 \mathrm{~W}$ at $1 \% \mathrm{O}_{2} / \mathrm{Ar}$, (v) $300 \mathrm{~W}$ at $2 \% \mathrm{O}_{2} / \mathrm{Ar}$, (vi) $200 \mathrm{~W}$ at $0.2 \% \mathrm{O}_{2} / \mathrm{Ar}$, (vii) $100 \mathrm{~W}$ at $0.2 \% \mathrm{O}_{2} / \mathrm{Ar}$ and (viii) $40 \mathrm{~W}$ at $0.2 \%$ $\mathrm{O}_{2} /$ Ar. The deposition of these films took place in close succession and when the target was new to avoid the unfavourable effects of energetic negative oxygen ion impaction in the growing film that become increasingly influential on the thin film properties with racetrack formation ${ }^{13}$. The deposition rate for the (ii) and (iii) films, was $16 \pm 0.2 \mathrm{~nm} / \mathrm{min}$ as ascertained from TEM analysis. Overall the deposition rate was mostly dependant on the rf power and a little on the oxygen flow rate ratio used in this study.

Tables 1 and 2 show the increase in mobility for non-aged (ii) and (vi) films following laser annealing in air at $248 \mathrm{~nm}$ at two levels of fluence; medium [83 and $\left.86 \mathrm{~mJ} / \mathrm{cm}^{2}\left( \pm 1.5 \mathrm{~mJ} / \mathrm{cm}^{2}\right)\right]$ and low $[51$ and $\left.52 \mathrm{~mJ} / \mathrm{cm}^{2}\left( \pm 1.5 \mathrm{~mJ} / \mathrm{cm}^{2}\right)\right]$. Higher levels of fluence $\left(100-295 \mathrm{~mJ} \mathrm{~cm}{ }^{-2}\right)$ were also investigated but the resultant films were very resistive with values outside the measuring range of the four point probe apparatus. High laser fluence annealing resulted in some samples undergoing ablation and subsequently acquiring a hazy appearance with a rough surface (evident from AFM imaging) while some others did not show any visible change but the measured transmittance was about $5 \%$ reduced. Hence, in order to investigate the effects of higher total laser irradiation dose, multiple lower fluence pulses were used in the range of $200-6000$ pulses.

The highest mobility value for the non-aged samples following laser processing was $15.9 \mathrm{~cm}^{2} \mathrm{~V}^{-1} \mathrm{~s}^{-1}$ (representing a 12 fold increase). The sample was sourced from the (vi) film (deposited at 200 $\mathrm{W} / 0.2 \% \mathrm{O}_{2} / \mathrm{Ar}$ ) and was processed at $86 \mathrm{~mJ} \mathrm{~cm}^{-2}$ and 1000 pulses. 
For both medium and low laser fluence the carrier concentration increases substantially following the first dose of 200 pulses but remains almost constant for the subsequent incremental doses indicating that the observed increase in mobility is not due to carrier density increase $\mathrm{e}^{14}$.

The carrier concentration increases in accordance to the initial carrier concentration of the asdeposited sample. Hence the laser processed samples from film (vi) had overall larger carrier concentrations than the (ii) samples.

The main origin for the observed difference in carrier concentration of the as-deposited $\mathrm{ZnO}$ films might be the quantity of oxygen present in the sputtering gas during the deposition of the various films. Defect generation due to oxygen species bombardment of the growing film with higher oxygen concentrations in the sputtering gas coupled with high rf power should also be considered as an additional cause for the observed carrier concentration differences. On the other hand, the difference in Hall mobility is due to different grain boundary defect state densities. This is exemplified in the drastic reduction of Hall mobility when using a high rf power (300 W) compared to a lower rf power $(200 \mathrm{~W})$ for the same oxygen flow rate percentage $(0.2 \%)$. The increase in rf power is expected to result in a more disordered lattice. The Hall mobility for the as-deposit samples from films (vi) and (i) was $2.1 \mathrm{~cm}^{2} \mathrm{~V}^{-1} \mathrm{~s}^{-1}$ and $0.05 \mathrm{~cm}^{2} \mathrm{~V}^{-1} \mathrm{~s}^{-1}$ respectively.

At medium fluence the mobility of the (ii) samples decreases steadily with increasing dose after reaching a maximum value while the (vi) samples become highly resistive and not possible to measure using the Hall system, when more than 1000 pulses are used.

Table 1 Hall mobility and carrier concentration of the samples from the (ii-300 W-0.5\% $\mathrm{O}_{2}$ ) and (vi$\left.200 \mathrm{~W}-0.2 \% \mathrm{O}_{2}\right)$ films measured at room temperature at medium and low laser fluence annealed immediately after deposition.

\begin{tabular}{|l|l|l|l|}
\hline \multicolumn{3}{|c|}{ (ii) $300 \mathrm{~W}-0.5 \% \mathrm{O}_{2} / \mathrm{Ar}$} \\
\hline Hall mobility $\left(\mathrm{cm}^{2} \mathrm{~V}^{-1} \mathrm{~s}^{-1}\right)$ & bulk carrier concentration $\left(\mathrm{cm}^{-3}\right)$ & $\begin{array}{c}\text { Number of } \\
\text { pulses }\end{array}$ & Energy density $\left(\mathrm{mJ} \mathrm{cm}^{-2}\right)$ \\
\hline
\end{tabular}




\begin{tabular}{|c|c|c|c|}
\hline 2.13 & $1.30 \times 10^{18}$ & 0 & \\
\hline 5.22 & $1.64 \times 10^{19}$ & 200 & \multirow{2}{*}{} \\
\hline 7.40 & $1.56 \times 10^{19}$ & 500 \\
\hline 11.63 & $1.07 \times 10^{19}$ & 1000 \\
\hline 10.62 & $1.57 \times 10^{19}$ & 2000 & \multirow{2}{*}{} \\
\hline 9.36 & $1.33 \times 10^{19}$ & 3000 \\
\hline 8.52 & $1.99 \times 10^{19}$ & 4000 \\
\hline 4.55 & $6.50 \times 10^{18}$ & 6000 & \multirow{2}{*}{$\mathbf{5 2}$} \\
\hline 3.52 & $3.05 \times 10^{18}$ & 200 & \\
\hline 3.97 & $4.16 \times 10^{18}$ & 500 & \\
\hline 5.10 & $5.37 \times 10^{18}$ & 1000 \\
\hline 6.57 & $4.70 \times 10^{18}$ & 2000 \\
\hline 8.58 & $6.12 \times 10^{18}$ & 4000 \\
\hline
\end{tabular}

Table 2 Hall mobility and carrier concentration of the samples from the (vi-200 W-0.2\% $\left.\mathrm{O}_{2}\right)$ films measured at room temperature at medium and low laser fluence annealed immediately after deposition.

\begin{tabular}{|c|c|c|c|}
\hline \multicolumn{3}{|c|}{ (vi) $200 \mathbf{W}-\mathbf{0 . 2} \% \mathbf{O}_{2} / \mathbf{A r}$} \\
\cline { 1 - 3 } Hall mobility $\left(\mathbf{c m}^{\mathbf{2}} \mathbf{V}^{-1} \mathbf{s}^{-1}\right)$ & bulk carrier concentration $\left(\mathbf{c m}^{-3}\right)$ & $\begin{array}{c}\text { Number of } \\
\text { pulses }\end{array}$ & Energy density $\left(\mathbf{m J ~ c m}^{-2}\right)$ \\
\hline 1.35 & $1.17 \times 10^{19}$ & 0 & \\
\hline 9.57 & $3.36 \times 10^{19}$ & 200 & \multirow{2}{*}{$\mathbf{2}$} \\
\hline 12.72 & $3.08 \times 10^{19}$ & 500 & \multirow{2}{*}{$\mathbf{5 1}$} \\
\hline 15.96 & $3.45 \times 10^{19}$ & 1000 & \\
\hline 3.74 & $2.72 \times 10^{19}$ & 1000 & \\
\hline 7.99 & $1.53 \times 10^{19}$ & 2000 & \\
\hline 8.55 & $1.62 \times 10^{19}$ & 4000 & \\
\hline
\end{tabular}

As we suggest in later sections, predominantly grain boundary modification is the possible cause of the observable mobility increase due to a reduction of defects at grain boundaries. It is not simply the result of barrier height reduction with carrier density increase due to filling up of grain boundary defect states ${ }^{14}$. This hypothesis is supported by the fact that two (ii) samples annealed at medium and low fluence using 4000 and 6000 pulses respectively, have similar mobility $\left(\sim 8.5 \mathrm{~cm}^{2} \mathrm{~V}^{-1} \mathrm{~s}^{-1}\right)$ while they also have substantially different carrier densities (i.e. differing by 3.3 fold).

\subsection{Room temperature Hall effect measurements - Aged samples}


Samples from all films were aged for 20 - 25 months in the laboratory $\left(20{ }^{\circ} \mathrm{C}-28{ }^{\circ} \mathrm{C}\right.$ with $25 \%-35$ $\%$ relative humidity) stored in closed petri-dishes. The films were exposed to fluorescent light during this period at an average illuminance of 120 lux.

The resistivity status of the films does not change with aging, and the resistive films remain resistive. They were subsequently annealed at medium fluence using multiple pulses. However, in contrast to the non-aged films, laser annealing lowered significantly the resistivity in most aged films.

Table 3 shows the optimised processing conditions for laser annealed samples from films aged in air that attained the highest mobility.

It was not possible to produce conductive samples from the aged films deposited at low rf sputtering power (40 W and $100 \mathrm{~W}$ ) which remain highly resistive after laser processing. Nano-indentation experiments carried out on aged (i), (iii), (v) and (viii) films showed that the (viii - $40 \mathrm{~W} / 0.2 \% \mathrm{O}_{2} / \mathrm{Ar}$ ) was quite more compact compared to the rest that were grown using $300 \mathrm{~W}$ rf power at different oxygen concentrations. Hence it seems that the (viii) was not affected much by aging. Confirmation of the above results was achieved by estimating the etching time using argon ions in an XPS system monitoring the silicon content to identify the glass/ZnO interface. The etching rate of the aged film grown at $40 \mathrm{~W}$ was more than five times longer than the aged films gown at $300 \mathrm{~W}$. We can ascribe this difference to the low deposition rate of the (viii) film $(1.8 \mathrm{~nm} / \mathrm{min}$ compared to about $18 \mathrm{~nm} / \mathrm{min}$ for the films grown at $300 \mathrm{~W}$ ).

The carrier concentration initially increases with the number of pulses. Any further increase in the number of pulses causes the carrier concentration to decrease as shown in Table 4 . The initial increase in carrier concentration is an indication of elimination of charge auto-compensation defects while the decrease is an indication of reduction of point defects in the intra-grain regions. The mobility keeps increasing with the number of pulses which is an indication of reduction of defect states at the grain boundaries. 
Table 3 shows the mobility, carrier concentration and number of pulses at medium fluence (83- 86 $\mathrm{mJ} / \mathrm{cm}^{2}$ ) required to produce the highest mobility by laser annealing at medium fluence for aged films.

The sputtering conditions are shown in the last two columns.

\begin{tabular}{c|ccc}
\hline Hall mobility $\left(\mathbf{c m}^{2} \boldsymbol{V}^{-1} \boldsymbol{s}^{-1}\right)$ & bulk carrier concentration $\left(\mathbf{c m}^{-3}\right)$ & $\begin{array}{c}\text { Number of laser } \\
\text { pulses }\end{array}$ & $\begin{array}{c}\text { Sputtering } \\
\text { conditions }\end{array}$ \\
\hline 15.37 & $1.90 \times 10^{19}$ & 6000 & $(\mathrm{ii})-300 \mathrm{~W}-0.5 \% \mathrm{O}_{2} / \mathrm{Ar}$ \\
17.30 & $2.30 \times 10^{18}$ & 3000 & $\left(\right.$ (iii) $-300 \mathrm{~W}-0.7 \% \mathrm{O}_{2} / \mathrm{Ar}$ \\
13.65 & $1.29 \times 10^{19}$ & 2000 & $(\mathrm{iv})-300 \mathrm{~W}-1 \% \mathrm{O}_{2} / \mathrm{Ar}$ \\
1.40 & $8.75 \times 10^{18}$ & 40 & $(\mathrm{v})-300 \mathrm{~W}-2 \% \mathrm{O}_{2} / \mathrm{Ar}$ \\
1.56 & $7.81 \times 10^{18}$ & 260 & $(\mathrm{vi})-200 \mathrm{~W}-0.2 \% \mathrm{O}_{2} / \mathrm{Ar}$ \\
\hline
\end{tabular}

Table 4 shows the mobility, carrier concentration and number of pulses at medium fluence for increasing number of pulses for the films (iv) and (iii).

\begin{tabular}{c|ccc}
\hline Hall mobility $\left(\mathbf{c m}^{2} \boldsymbol{V}^{-1} \boldsymbol{s}^{-1}\right)$ & bulk carrier concentration $\left(\boldsymbol{c m}^{-3}\right)$ & $\begin{array}{c}\text { Number of laser } \\
\text { pulses }\end{array}$ & $\begin{array}{c}\text { Sputtering } \\
\text { conditions }\end{array}$ \\
\hline 4.96 & $8.88 \times 10^{18}$ & 400 & (iv)-300W- $1 \% \mathrm{O}_{2} / \mathrm{Ar}$ \\
7.62 & $1.59 \times 10^{19}$ & 1000 & (iv)-300W- $1 \% \mathrm{O}_{2} / \mathrm{Ar}$ \\
13.65 & $1.29 \times 10^{19}$ & 2000 & (iv)-300W- $1 \% \mathrm{O}_{2} / \mathrm{Ar}$ \\
12.38 & $1.09 \times 10^{19}$ & 3000 & (iv)-300W- $1 \% \mathrm{O}_{2} / \mathrm{Ar}$ \\
1.90 & $6.00 \times 10^{18}$ & 500 & (iii)-300W- $0.7 \% \mathrm{O}_{2} / \mathrm{Ar}$ \\
13.10 & $1.70 \times 10^{19}$ & 1000 & (iii)-300W- $0.7 \% \mathrm{O}_{2} / \mathrm{Ar}$ \\
17.30 & $2.30 \times 10^{18}$ & 3000 & (iii)-300W- $0.7 \% \mathrm{O}_{2} / \mathrm{Ar}$ \\
\hline
\end{tabular}

Laser annealing following aging results in an overall higher mobility and relatively low carrier concentration in comparison to the non-aged films that were laser processed immediately after deposition. It is also evident that the deposition conditions (the $\mathrm{rf}$ sputtering power and the oxygen/argon flow rate ratio) are important factors that determine the effectiveness of laser annealing in producing films with high mobility and low carrier concentration.

In parallel to laser annealing, furnace thermal annealing in air, at $400^{\circ} \mathrm{C}$ and $500^{\circ} \mathrm{C}$ for $30-60 \mathrm{~min}$, was also investigated. However all thermally processed films were highly resistive even after aging. There are contrasting reports in the literature about the effects of thermal annealing on $\mathrm{ZnO}$ resistivity. Thermal annealing in air either reduces substantially the $\mathrm{ZnO}$ resistivity ${ }^{15}$ or increases the $\mathrm{ZnO}$ resistivity ${ }^{16,17}$. Desorption of oxygen is correlated with decrease in resistivity ${ }^{15}$ while absorption 
of oxygen with increase ${ }^{16}$. It has been postulated that electrical properties change with oxygen absorption along grain boundaries ${ }^{19}$. Hence it is plausible that grain boundary scattering is the limiting mechanism in thermal annealing.

\subsection{Variable temperature Hall effect measurements for as-deposited and aged films}

Variable temperature Hall measurements in the range of $78 \mathrm{~K}-360 \mathrm{~K}$ were used to study the conduction mechanism in selected laser-annealed samples (after deposition and after aging).

All samples showed a positive temperature coefficient of mobility, as shown in Figure 1, indicating that the conduction is governed by temperature-dependent activation processes like thermionic emission or thermionic field emission across grain boundaries ${ }^{14,18,19}$.

At low temperatures the mobility is independent of temperature when tunnelling across grain boundaries is predominant over temperature-activated processes ${ }^{19}$. Hence for the results presented here, at low temperatures the investigated films showed a mixed conduction of tunnelling and temperature activation similarly to other studies ${ }^{18,19}$.

Figure 2 shows the variation of carrier concentration with temperature for the samples corresponding to Figure 1.

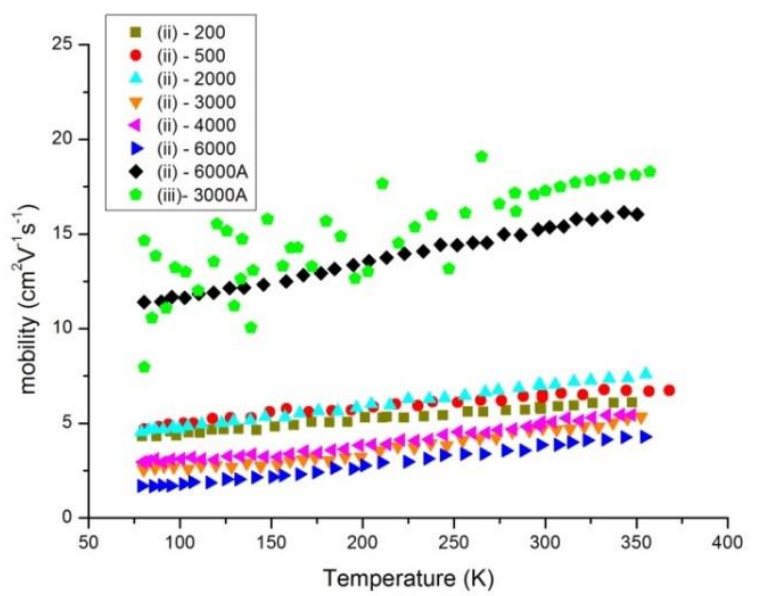

Figure 1 Temperature variation of mobility with laser processing for samples from films (ii-300 W$\left.0.5 \% \mathrm{O}_{2}\right)$ and (iii-300 $\mathrm{W}-0.7 \% \mathrm{O}_{2}$ ). The numbers indicate the number of laser pulses used. 'A' 
indicates the two samples from films (ii) and (iii) that were laser-annealed after aging and stands for 'Aged'.

The non-aged samples from film (ii), annealed up to 2000 pulses, showed a positive temperature coefficient of carrier concentration in the whole investigated temperature range. A thermally activated process is imposed because the trapped carrier concentration in grain boundaries is smaller than the free carrier concentration in the grain interior causing the depletion layer width to vary substantially with temperature.

The non-aged samples, from the (ii) film, annealed at 3000, 4000 and 6000 pulses showed a different behaviour. The first two showed a positive temperature coefficient of carrier concentration at low temperatures and a small negative temperature coefficient at high temperatures. The sample annealed at 6000 pulses showed almost no variation of carrier concentration in the low temperature range while it has a pronounced negative temperature coefficient in the high temperature range.

It is evident that these three films have distinct behaviour i.e. in the range $150 \mathrm{~K}-300 \mathrm{~K}$. As the temperature is lowered the carrier concentration is increasing indicating that electrons are emitted into the conduction band. The origin of carrier increase is likely to be acceptor states at the grain boundaries. Acceptor states are negatively charged when occupied and neutral when empty releasing their electrons into the conduction band as the Fermi level moves away from the conduction band when the temeprature is increasing ${ }^{20,21}$. When they become exhausted normal thermionic activated conduction starts as for the 3000 and 4000 pulses samples. However, for the 6000 pulses sample the acceptor state density is large and carrier emission does not stop as the temperature is increased. The release of electrons occurs now at a constant rate. 


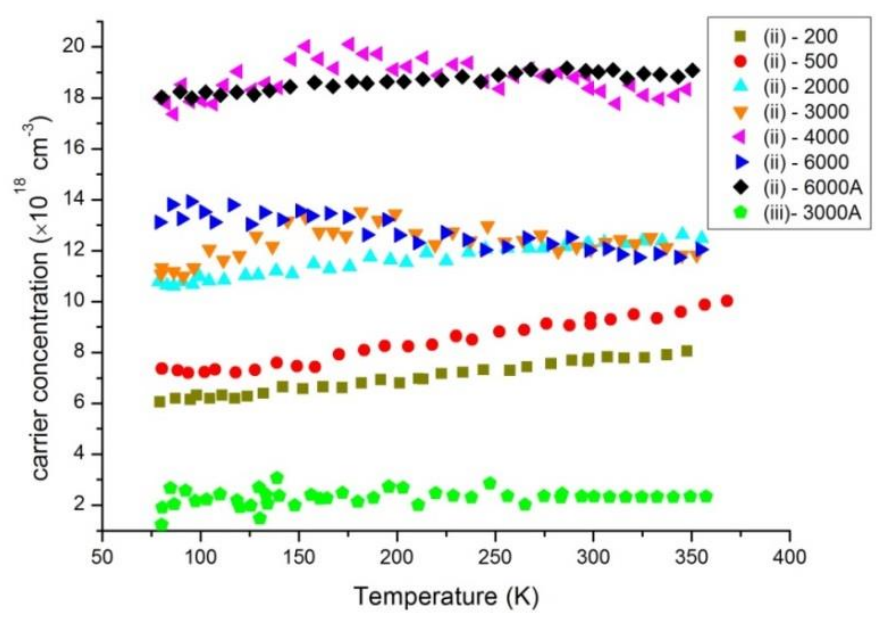

Figure 2 Temperature dependence of carrier concentration with laser processing for samples from films (ii-300 W-0.5\% $\mathrm{O}_{2}$ ) and (iii-300 W-0.7\% $\mathrm{O}_{2}$ ). The numbers indicate the number of laser pulses used. A indicates the two samples from films (ii) and (iii) that were laser-annealed after aging and stands for 'Aged'.

The aged sample from film (ii) shows a smaller positive temperature coefficient at the high end of the temperature range than the lower end. This behaviour is exemplified in the aged sample (iii)-3000A. This sample shows a temperature-independent carrier concentration response with temperatures between $210 \mathrm{~K}$ and $360 \mathrm{~K}$, and a small positive temperature coefficient between $76 \mathrm{~K}$ and $210 \mathrm{~K}$. A usual explanation for a temperature-independent carrier concentration is that a degenerate semiconductor (which is not the case here) or a tunnelling process is involved. When the total number of trapped carriers at grain boundaries is considerably smaller than the number of free carriers in the grain interior the grain boundary depletion widths are thin and the measured carrier concentration is comparable to the carrier concentration in the grain interior ${ }^{22}$. As the temperature decreases the free carrier concentration in the grain interior decreases and the depletion widths become larger. It is expected that the carrier conduction will then become thermally activated ${ }^{18,22}$. In the higher temperature range $(210 \mathrm{~K}-360 \mathrm{~K})$ a depletion region is also formed but because the carrier concentration is independent of temperature it is expected to be quite thin. The (iii)-3000A film showed the highest overall mobility value. This is among the highest Hall mobility values in the literature for a $\mathrm{ZnO}$ film processed at ambient temperature $\left(\mathrm{T}<70{ }^{\circ} \mathrm{C}\right)$ having simultaneously low 
carrier concentration which is essential for the ON/OFF ratio of a TFT. Few reports exist of ZnO thin films with a simultaneous high Hall mobility and low carrier concentration at low temperatures and on amorphous substrates ${ }^{23,24}$. Notably, $\mathrm{ZnO}$ thin films (30 nm thick) deposited by filtered cathodic arc deposition, at $110^{\circ} \mathrm{C}$, with similar electrical properties $\left(\mu=17.3 \mathrm{~cm}^{2} \mathrm{~V}^{-1} \mathrm{sec}^{-1}\right.$ and $\left.\mathrm{n} \sim 2 \times 10^{18} \mathrm{~cm}^{-3}\right)$ have been used successfully as active layers in TFT fabrication on transparent and flexible substrates $^{23}$. In a well known study by Ellmer ${ }^{14}$ an empirical graph of the Hall mobility vs carrier concentration was presented that collated a multitude of literature data of intrinsic and doped $\mathrm{ZnO}$ films. The films were deposited on float glass and sapphire substrates using (dc or $\mathrm{rf}$ ) magnetron sputtering and pulse laser deposition (PLD). By fitting the empirical trend with the barrier-limited transport model of Seto $^{25}$ they extracted high values for the defect state density at the grain boundaries. Extrapolation of the fitting routine to low carrier concentrations $\left(\mathrm{n}<10^{18} \mathrm{~cm}^{-3}\right.$ ), for the lower values of the defect state density, predicted Hall mobility values around $1 \mathrm{~cm}^{2} \mathrm{~V}^{-1} \mathrm{~s}^{-1}$. The importance of the grain boundary defect state density is exemplified by the Hall properties of high quality heteroepitaxial $\mathrm{ZnO}$ film grown by $\mathrm{PLD}$ at high substrate temperatures $\left(\sim 750{ }^{\circ} \mathrm{C}\right)$ on sapphire substrates. These films show high scattering of Hall mobility values $\left(\sim 1 \mathrm{~cm}^{2} \mathrm{~V}^{-1} \mathrm{~s}^{-1}-155 \mathrm{~cm}^{2} \mathrm{~V}^{-1} \mathrm{~s}^{-1}\right)$ for low carrier concentrations $\left(10^{16} \mathrm{~cm}^{-3}-10^{18} \mathrm{~cm}^{-3}\right)$ due to various degrees of the barrier layer quality, during its optimisation development, that is used to template the overgrowing $\mathrm{ZnO}$ films ${ }^{26}$.

\subsection{Characterisation using TEM, AFM and Optical spectrophotometry.}

\subsubsection{TEM and AFM}

Cross-sectional TEM investigation (Figure 3) of five samples from the (ii) film was carried out: three of the samples were annealed at medium fluence using 0, 500 and 6000 pulses, one at low fluence using 6000 pulses and, an aged sample at medium fluence and 6000 pulses. As discussed before, the aged sample showed the highest mobility observed in samples from film (ii). 

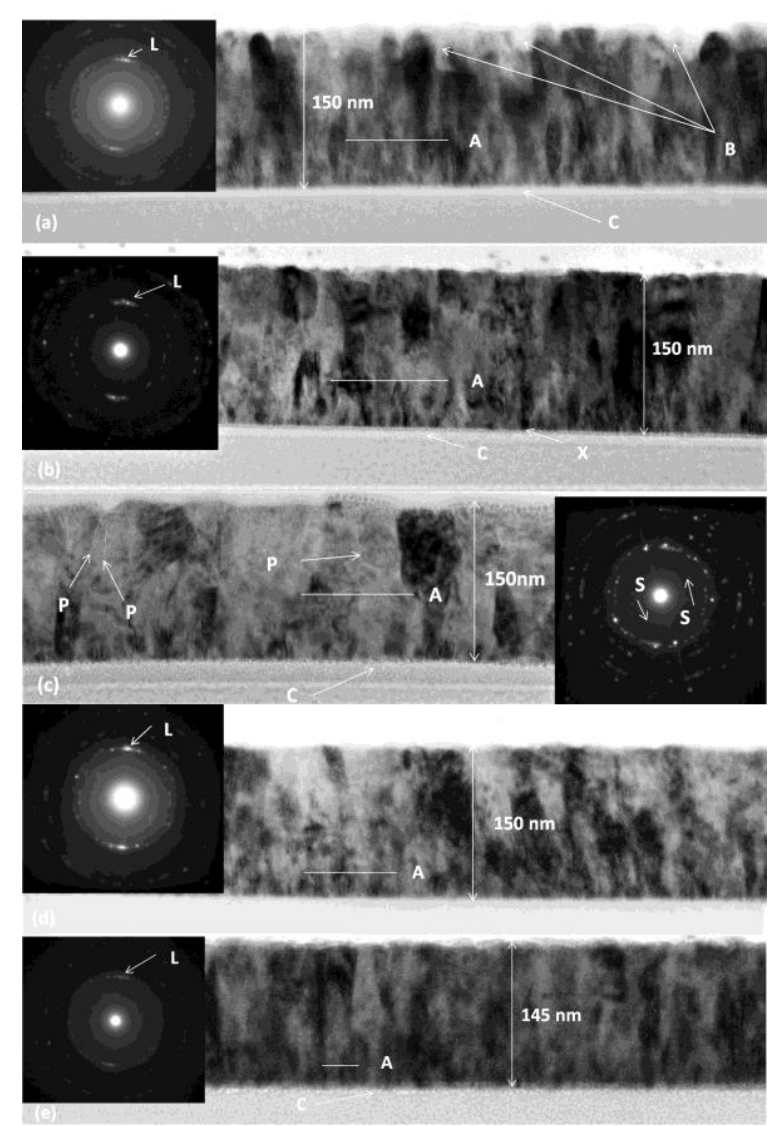

Figure 3 Cross-sectional TEM of samples from film (ii-300 W/O.2\% $\mathrm{O}_{2}$ ) laser-annealed at medium fluence and (a) 0,(b) 500,(c) 6000 pulses after deposition, (d) was aged before laser annealing at 6000 pulses while, (e) was laser-annealed at low fluence and 6000 pulses immediately after deposition.

It is well known that in the rf sputtering process the initial deposited layers are characterised by amorphous regions and/or small crystal grains which they grow into bigger grains ${ }^{27}$ as the film thickness increases. This is evident in the double layer structure that is demarcated by A in Figure 3(a) dividing the total film thickness into bottom and top layers with different average grain sizes. This double layer structure is evidenced in all (ii) samples investigated by TEM as labelled by A. Surface roughness is shown as $\mathrm{B}$. The thin layer of fine particulate material at $\mathrm{X}$ and the mixed phase of $\mathrm{ZnO}$ and glass at $\mathrm{C}$ might also be products of the high $\mathrm{rf}$ power used. The band at $\mathrm{C}$ depends also on the total laser energy used and it becomes larger as more pulses are used. 
For the laser-annealed non-aged sample i.e. processed immediately after deposition at high number of pulses, the bottom layer width grows larger as shown in Figure 3(c). However the non-aged sample processed at low fluence and the aged sample processed at medium fluence have a more uniform cross-sectional structure. Furthermore the bottom layer, at the height A, in these two samples is smaller in width.

In Figure 3 the inset in each image shows the selected area electron diffraction (SAED) pattern demonstrating formation of hexagonal phase from the (002) reflection (at L) for all samples apart from sample (c) annealed at medium fluence and 6000 pulses. The last shows absence of texturing and formation of a secondary phase at S, as shown in the SAED pattern, due to large total energy used in processing. The secondary phase diffraction might be originating from the low density layer at $\mathrm{C}$ ( $8.5 \mathrm{~nm}$ thick). The phase at $\mathrm{C}$ is almost absent in the sample annealed at low fluence and, completely absent in the aged sample annealed at medium fluence. The last two samples were annealed both at high number of pulses (6000). Furthermore there is also evidence of lack of diffraction at $\mathrm{P}$ along some grain boundaries at the top half of the film showing in the image as white colour and might originate from porosity or amorphous material formed along some grain boundaries. Figure 4 shows a HAADF (High Angle Annular Dark Field) image from an aged sample from film (iii - 300 W/0.7\% $\left.\mathrm{O}_{2}\right)$ that was laser annealed using medium fluence $\left(83 \mathrm{~mJ} \mathrm{~cm}^{-2}\right)$ and 3500 pulses. The sample is very resistive and the amorphous areas are shown as black. These areas are observed along some grain boundaries. EELS (Electron Energy Loss Spectroscopy) performed along one such grain boundary proved that the amorphous material is made solely from zinc oxide. Hence the observation at $\mathrm{P}$, in Figure $3 \mathrm{c}$, is likely to be amorphous zinc oxide. Both the amorphous material at $\mathrm{P}$ and the band of low density material at $\mathrm{C}$ are the results of laser irradiation.

From the information of Figure 3, the widths of the bottom and top layer as well as the grain sizes in these layers for the various samples sourced from (ii) film are shown in Table 5. There is almost no change of the average grain size for laser processed sample at low fluence indicating that the change observed in mobility and carrier concentration at low fluence originates mainly from grain boundary modification. Additional support to this argument is offered by the changes in mobility after medium 
fluence anneal of an as-deposit and an aged sample using high number of pulses (6000 pulses). There is substantial top layer grain growth in both of these films but only the aged sample shows increased mobility. The mobility in the as-deposit samples continuously decreases after the optimised number of pulses is reached and at 6000 pulses the mobility attains its smallest value.

From the current TEM study there is no strong evidence that the crystallographic defect contents of the five oxide layers are much different to each other.

To investigate further the surface topology and the impact of laser processing AFM analysis was carried out. AFM analysis on selected samples from the (iii-300 W/0.7\% $\mathrm{O}_{2}$ ) film showed that the as deposited sample presents a low RMS roughness $(1.69 \mathrm{~nm})$, the laser annealed sample using medium fluence leads to an increase in the RMS roughness $(4.19 \mathrm{~nm})$ while laser annealing at low fluence creates surfaces with RMS roughness of $2.8 \mathrm{~nm}$. There is considerable melting at medium fluence which is evident by the creation of ripples and corrugations on the surface ${ }^{28}$. The surface at low fluence is more uniform and rippling is absent as is evident from Figure 5.

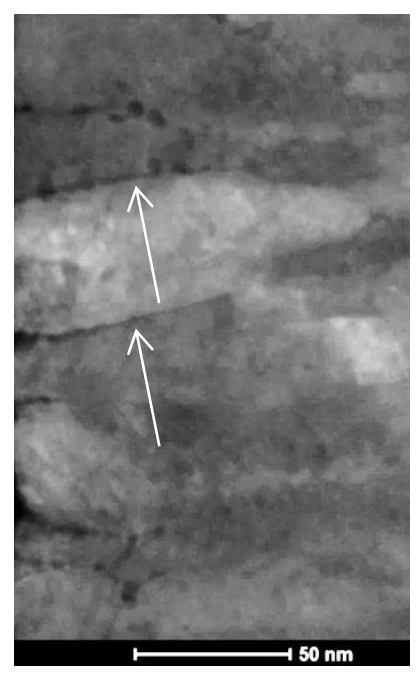

Figure 4 Cross-sectional HRTEM (HAADF image) section of an aged sample from film (iii-300 W/0.7\% $\mathrm{O}_{2}$ ) annealed at medium fluence and 3500 pulses showing amorphous zinc oxide been formed along some grain boundaries as shown with the arrows. 
Table 5 Widths of the bottom and top layer as well as the grain sizes in these layers for the various samples sourced from (ii) film.

\begin{tabular}{|c|c|c|c|c|c|}
\hline & \multirow[t]{2}{*}{$\overline{\text { As-Deposited }}$} & \multicolumn{3}{|c|}{$83 \mathrm{~mJ} \mathrm{~cm}^{-2}$} & \multirow{2}{*}{$\begin{array}{l}52 \mathrm{~mJ} \mathrm{~cm}^{-2} \\
6000 \text { pulses }\end{array}$} \\
\hline & & 500 pulses & 6000 pulses & 6000 pulses (aged) & \\
\hline Top Layer Thickness (nm) & $120-150$ & 120 & 75 & 135 & 140 \\
\hline Bottom Layer Thickness (nm) & $0-30$ & 30 & 75 & 10 & 10 \\
\hline Top Average Grain width (nm) & 27 & 27 & 46 & 41 & 25 \\
\hline Bottom Average Grain width (nm) & 13 & 13 & 21 & 15 & 17 \\
\hline
\end{tabular}

Amorphisation of grain boundaries has been demonstrated to take place in polycrystalline solids with highly constrained grain boundaries ${ }^{29,30}$ in order to minimise the free energy of the solid during sintering ${ }^{31}$. The driving force for this transformation is the high energy of amorphous grain boundaries in relation to the grain interior ${ }^{32}$ which can lead to enhanced diffusion and enlargement of grain boundaries $^{33}$.
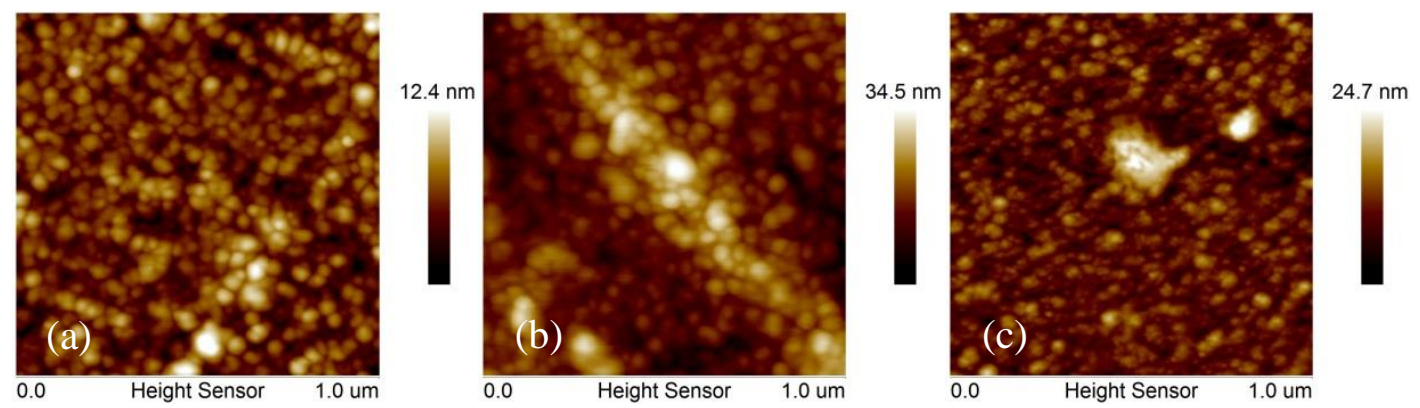

Figure 5 2D AFM images of an aged as-deposit (iii) sample shown in (a) and (d), a laser annealed sample at medium fluence and 3000 pulses i.e. (iii)-3000A shown in (b) and (e) and a laser annealed sample at low fluence $\left(60 \mathrm{~mJ} \mathrm{~cm}^{-2}\right.$ and 3000 pulses. 
It is also possible that hydroxylic groups can provide additional driving force ${ }^{34}$ for densification at lower temperatures under high heating rates as there is evidence of some amorphisation of the top layers as well.

\subsubsection{Optical spectrophotometry}

Structural information from TEM can be complemented by optical absorption characteristics of the optically active defects in the investigated films. With TEM analysis was not possible to differentiate between the non-aged and, aged films before any laser processing took place. Absorption in the 400 $\mathrm{nm}-1000 \mathrm{~nm}(3.1 \mathrm{eV}-1.24 \mathrm{eV})$ region is attributed to transitions in the localised states in the forbidden band gap that are originated from intrinsic lattice defects such as oxygen vacancies, zinc interstitials etc ${ }^{35,36}$. The transmittance spectra in the visible region of the two conditions show distinct dissimilarities that have been induced by the aging process as can be seen in Figure 6. There is increase in transmittance in the long wavelengths and shifting of the absorption edge towards longer wavelengths. Transmittance increases in the long wavelengths can be due to smaller absorption but also due to decrease of free carrier concentration. Red shifting of the absorption edge is an indication of increased band tailing due to defect generation ${ }^{37,38}$. The transmittance of aged and laser annealed samples from (ii) and (iii) films that showed largest mobility after laser processing are also depicted with green lines.

Due to small size of the processed samples, necessitated by the laser beam size, it was not possible to measure reflectance and hence to acquire the absorption coefficient. However, it is evident that the absorption edge for these two films has moved to shorter wavelengths which is an indication of a larger energy band gap. Similar blue shift of the absorption edge is associated with increase in bandgap due to decrease of band tailing width ${ }^{39}$ and it is not due to Berstein-Moss effect as all of these films are non-degenerate. 

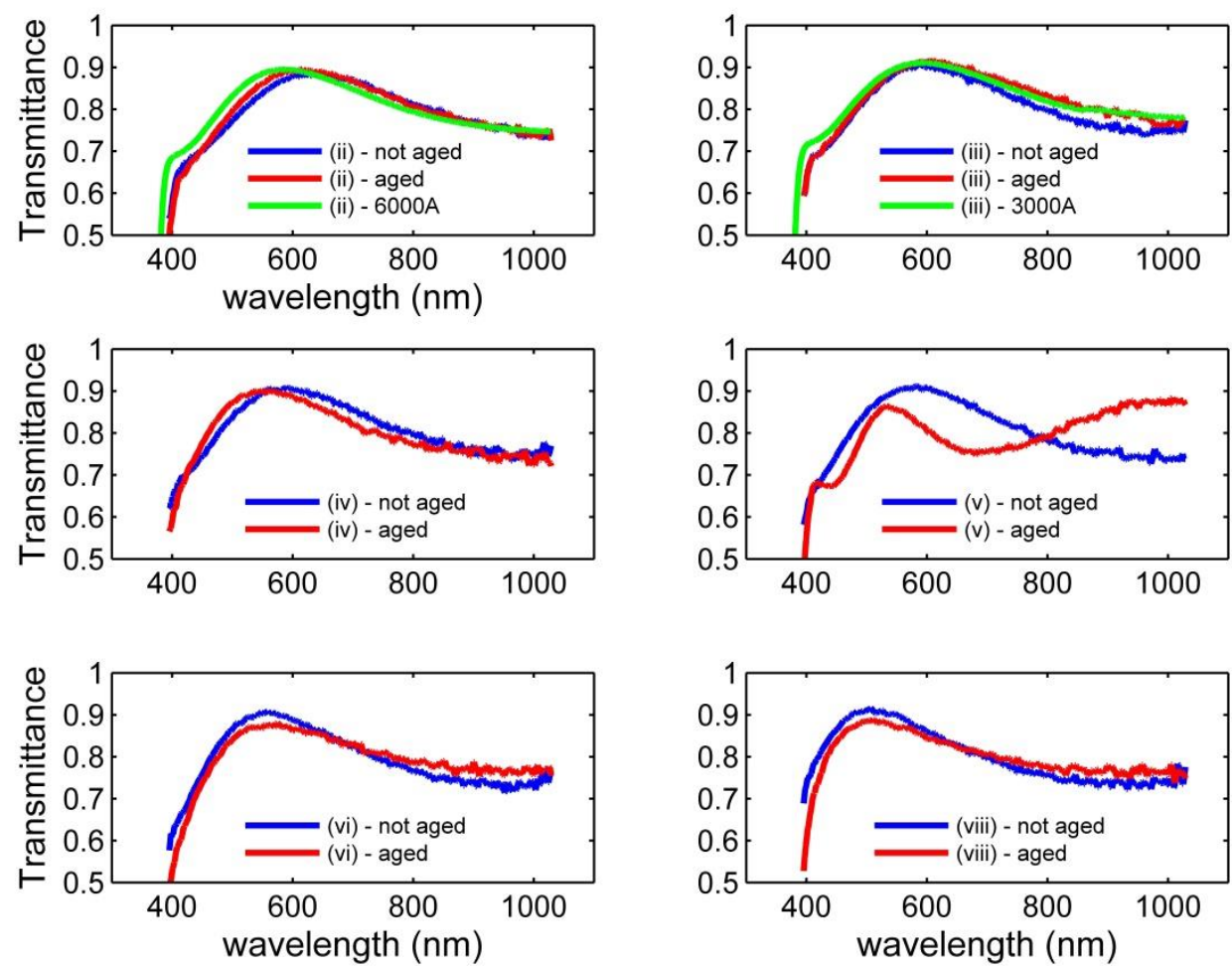

Figure 6 Transmittance spectra of not aged and agent as-deposited samples from films (ii-300 $\left.\mathrm{W} / 0.5 \% \mathrm{O}_{2}\right),\left(\mathrm{iii}-300 \mathrm{~W} / 0.7 \% \mathrm{O}_{2}\right),\left(\mathrm{iv}-300 \mathrm{~W} / 1 \% \mathrm{O}_{2}\right),\left(\mathrm{v}-300 \mathrm{~W} / 2 \% \mathrm{O}_{2}\right),\left(v i-200 \mathrm{~W} / 0.2 \% \mathrm{O}_{2}\right)$ and $(v i i i-$ $\left.40 \mathrm{~W} / 0.2 \% \mathrm{O}_{2}\right)$. The green lines show the transmittance spectra of aged and laser-annealed samples from (ii) and (iii) films presented in tables 1 and 2. The numbers in the legend depict the number of pulses used and 'A' stands for aged. The TEM of the (ii)-6000A was presented in Figure 3(c).

It is also evident that the (v) film (i.e. $300 \mathrm{~W} / 2 \% \mathrm{O}_{2}$ ) experiences the greatest change in transmittance after aging which might be attributed to the disrupted and defective structure of the film caused by the destructive bombardment of the negative oxygen ions ${ }^{40}$ and increase of absorption due to generation of various defects ${ }^{35}$. The transmittance at longer wavelengths is also distinctly increased after aging which might be an indication of free carrier concentration decrease ${ }^{41}$. This behaviour can be attributed to the reduction of oxygen vacancies that act as donors in $\mathrm{ZnO}$ films, due to absorption of oxygen 
species from the environment. Similar changes in transmittance have been observed in $\mathrm{ZnO}$ films of equivalent thickness after damp heat tests ${ }^{42,43}$ which are followed by structural changes.

It is well established that $\mathrm{ZnO}$ films during damp heat stability experiments absorb various oxygen, water and carbon dioxide mainly through grain boundaries ${ }^{44,45}$ that can diffuse to the inside of the grains if porosity is present ${ }^{46}$. Heating selected aged samples on a hotplate in air at $150{ }^{\circ} \mathrm{C}$ for $10 \mathrm{~min}$ did not revert the changes observed in transmittance which implies that a permanent structural alteration has occurred due to aging.

Figure 7 shows the absorption coefficient for selected laser processed samples from films (ii) and (iii).

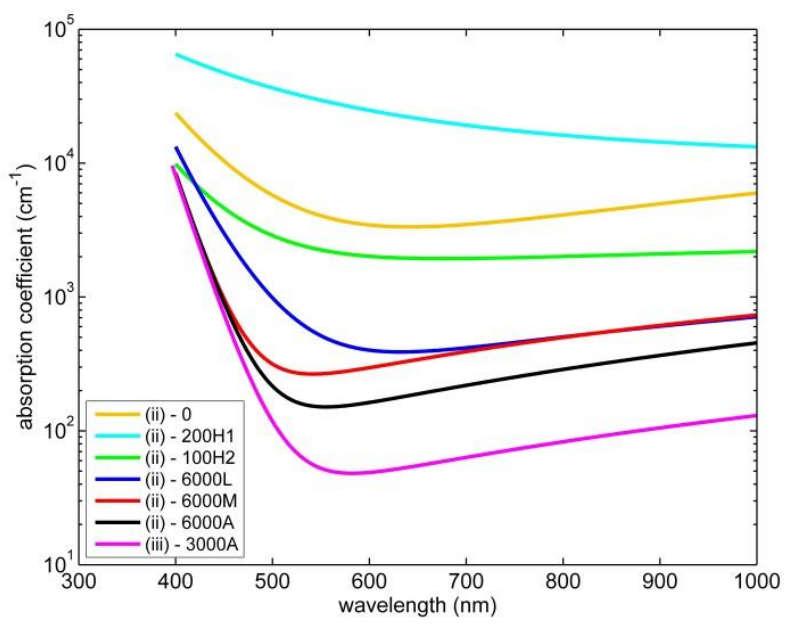

Figure 7 The optical absorption coefficient for selected samples from (ii-300 W/0.5\% $\mathrm{O}_{2}$ ) and (iii-300 W/0.7\% $\mathrm{O}_{2}$ ) films using various levels of fluence and number of pulses. The letters denote the fluence level ( $\left.L=52 \mathrm{~mJ} \mathrm{~cm}^{-2}, M=83 \mathrm{~mJ} \mathrm{~cm} \mathrm{~cm}^{-2}, \mathrm{H1}=127 \mathrm{~mJ} \mathrm{~cm}^{-2}, \mathrm{H2}=106 \mathrm{~mJ} \mathrm{~cm}^{-2}\right)$ and whether the sample was aged (A). The aged samples were processed at medium fluence $\left(83 \mathrm{~mJ} \mathrm{~cm}^{-2}\right)$.

A commercial package (Scout) was used to provide the fitting to the optical data (Transmittance and Reflectance). The thin films were modelled by using a three layer structure to simulate the bottom layer, the top layer and the surface roughness of the samples following the TEM and AFM investigation. In order to get a close fit to the measured data a harmonic oscillator, the standard Drude model, the O'Leary-Jonhson-Lim (OJL) model ${ }^{39}$ and a dielectric constant were used to describe the 
various contributions to the dielectric function. The fit parameters in the OJL model are the gap energy, the tail state exponent (gamma- $\gamma_{\mathrm{C}}$ ), the overall strength of the transition and a decay parameter to reduce the imaginary part of the dielectric function to zero at high frequencies. The mean square deviation between the measured and simulated spectral values had a goodness of fit rating for all simulations ranging from good to excellent (i.e. values lower than 0.00009).

Gamma $\left(\gamma_{C}\right)$ is equivalent to the Urbach energy $\left(E_{0}\right)$ which is an indication of the cumulative effect of structural disorder, impurities and electron-phonon interactions due to existence of structural disorder ${ }^{47}$. Gamma $\left(\gamma_{\mathrm{C}}=\mathrm{E}_{0}\right)$ is given by the equation:

$e^{\frac{\left(E-E_{c}\right)}{\gamma_{c}}}$

To put into perspective the quality of the investigated films and the effect of laser processing we can compare the Urbach energy of various films as reported in the literature. Typical values of Urbach energy for good quality polycrystalline $\mathrm{ZnO}$ thin films deposited at $223^{\circ} \mathrm{C}$ on sapphire wafers is found to be $84 \mathrm{meV}$ that decreases to $44 \mathrm{meV}$ after thermal annealing at 800 for 3 hours $^{48}$. Deposition of $\mathrm{ZnO}$ on Eagle glass substrates at room temperature using sputtering results in an Urbach energy close to $100 \mathrm{meV}^{48}$. The Urbach energy is related to the optical properties but the physical scenario that it expresses is related to the transport properties of the electrons. These localised tail states act as electron traps centres that contribute to conduction through a multiple-trapping-and-release conduction mechanism (MTR) ${ }^{49}$, The distribution of these trap centres is due to the interface defects at the grain boundaries as well as the to the defects in the interior of the grains ${ }^{49}$.

The sharpness of the absorption profile near the UV and the decreasing value of $\gamma_{C}$ indicate the increase of band gap with number of pulses (ref). Gamma values for the samples in Figure 7 for the (i) $-0,200 \mathrm{H} 1,100 \mathrm{H} 2,6000 \mathrm{~L}, 6000 \mathrm{M}, 6000 \mathrm{~A}$ and (ii)-3000A samples are $\gamma_{\mathrm{C}}=312 \mathrm{meV}, 802 \mathrm{meV}, 329$ $\mathrm{meV}, 193 \mathrm{meV}, 128 \mathrm{meV}, 111 \mathrm{meV}$ and $91 \mathrm{meV}$ respectively. We also observe that the absorption in the long wavelengths becomes smaller with decreasing Urbach energy indicating progressive defect elimination.

\section{Discussion}


In $\mathrm{ZnO}$ the $248 \mathrm{~nm}$ energy is absorbed at the top $10 \mathrm{~nm}$ of the material ${ }^{50}$. The absorbed energy is subsequently converted to heat resulting in localised heating of the film surface. The heat spreads throughout the thin film by thermal conduction predicted to be carried solely by phonons for electron carrier concentrations smaller than ${ }^{51} 10^{19} \mathrm{~cm}^{-3}$. Since the irradiation is uniform across the sample's surface the heat spreads vertically and laterally. The equilibration of temperature is in the microseconds range ${ }^{52}$. Hence for a $10 \mathrm{~Hz}$ pulse repetition frequency temperature equilibration has been reached between successive pulses and temperature build up in the film is avoided.

From Table 5 we note that even after 500 pulses at medium fluence no significant structural change leading to noticeable average grain size growth occurred apart from a decreased surface roughness and a homogenised top layer. Various annealing studies of $\mathrm{ZnO}$ sputtered films indicate that structural modification, occasionally with concomitant alteration of the electrical properties, can occur as low as $200^{\circ} \mathrm{C}^{16,53,54,55,56}$.

During a single laser pulse the temperature change at the top surface layer follows Eq. (2) ${ }^{57}$.

$\Delta T\left({ }^{\circ} C\right)=\frac{2(1-R) J}{k}\left(\frac{D}{\pi t}\right)^{\frac{1}{2}}$

where $\mathrm{R}=0.16$ is the typical reflectivity at $248 \mathrm{~nm}$, $\mathrm{J}$ is the pulse energy per unit area, $\mathrm{k}$ is the thermal conductivity, $\mathrm{t}$ is the laser pulse duration and $\mathrm{D}$ is the thermal diffusivity. Published data were used for thermal conductivity and thermal diffusivity parameters ${ }^{58,59,60}$. Typical values for polycrystalline $\mathrm{ZnO}$ films range between $0.2-0.5 \mathrm{~W} \mathrm{~cm}^{-1} \mathrm{~K}^{-1}$. Typical thermal diffusivities range from $0.075 \mathrm{~cm}^{2} \mathrm{~s}^{-1}$ to $0.083 \mathrm{~cm}^{2} \mathrm{~s}^{-1}$. Hence, for one pulse at 83 or $52 \mathrm{~mJ} \mathrm{~cm}^{-2}$ with $\mathrm{k}=0.4 \mathrm{~W} \mathrm{~cm}^{-1} \mathrm{~K}^{-1}$ and $\mathrm{D}=0.075 \mathrm{~cm}^{2}$ $\mathrm{s}^{-1}$ a maximum top surface temperature is calculated at $\Delta \mathrm{T}=377^{\circ} \mathrm{C}$ or $236^{\circ} \mathrm{C}$ respectively.

The thermal conductivity is affected by intra-grained point defects but mainly depends on the disorder present in the grain boundaries due to scattering of phonons $s^{61,62,63,64}$. Hence thermal conductivity will be related mostly to the evolution of grain boundaries with laser dosage.

The TEM analysis revealed the different structures between the laser annealed non-aged and aged samples; a layer structure is distinct in the first while layering is almost absent in the second. TEM analysis also showed evidence of a low density material developing in the as deposit sample with 
increased total energy deposition but absent in the aged sample processed under the same laser conditions. Furthermore amorphisation of the grain boundaries, as is evidenced at $\mathrm{P}$ in Figure 3c, occurs for large laser energy dosage. The reason behind these observations might be due to thermal flow effects related to the particular phonon transport. Exposure of $\mathrm{ZnO}$ ceramic agglomerates to water vapour has been demonstrated to increase the thermal conductivity ${ }^{65}$. Hence the increased thermal conductivity of the aged sample, due to grain boundary modification with exposure, will present less resistance to the lateral transmission of phonons compared to the as-deposited sample. In the latter sample more heat will be directed along the grains interiors resulting in the modification of the substrate at $\mathrm{C}$. It is difficult to hypothesise about the nature of the defects in the grain boundaries of the as deposit sample and we cannot rule out that they are mainly intrinsic. According to Luckyanova and co-workers, the thermal conductivity of $\operatorname{Pr}_{0.1} \mathrm{Ce}_{0.9} \mathrm{O}_{2-\mathrm{d}}$ thin films can be tailored by changing the oxygen content of the films during deposition ${ }^{66}$. The thermal conductivity decreases with the in situ generation of oxygen vacancies ${ }^{66}$. Such intrinsic vacancies (zinc related vacancies and defect clusters) have been detected to accumulate at grain boundaries in polycrystalline $\mathrm{ZnO}$ films ${ }^{67}$. Generally grain boundaries due to their inherent disorder present an obstacle to phonon transport and this property is taken advantage of in the engineering of new thermoelectric materials ${ }^{68,69}$.

The significance of grain boundaries in the differing laser annealing behaviour of the aged films compared to the as-deposited films is evident from the above analysis and the experimental data presented in the previous sections. As noted above, it is very challenging to produce $\mathrm{ZnO}$ films with high mobility at the required carrier concentration range for TFT applications $\left(10^{17}-10^{18} \mathrm{~cm}^{-3}\right)$. It has been reported that even when the as-deposited films are of very high quality, $\left(107 \mathrm{~cm}^{2} \mathrm{~V}^{-1} \mathrm{~s}^{-1}\right.$ at $10^{14}$ $\left.\mathrm{cm}^{-3}\right)^{70}$ the carrier concentration requirements for TFT applications necessitate further processing which is most often thermal in nature resulting in introduction of defects with concomitant mobility reduction (i.e. $0.21 \mathrm{~cm}^{2} \mathrm{~V}^{-1} \mathrm{~s}^{-1}$ at $2 \times 10^{17} \mathrm{~cm}^{-3}$ after anneal in argon at $\left.300{ }^{\circ} \mathrm{C}\right)^{70}$. Oxygen, carbon dioxide or hydroxyl group physisorption and chemisorption, mainly through grain boundaries, might be the operant mechanism that promotes the high mobility during laser annealing of the aged films. The exact mechanism in not currently known but a slow diffusive process of air species seems to be 
the required condition. In damp heat experiments of conductive $\mathrm{ZnO}$ films, the resistivity increases with exposure time due to grain boundary absorption of environmental gasses that reduce the Hall mobility but leave unaffected the optical mobility ${ }^{71}$ that represents the mobility in the grain interiors. Adsorption of various gas species (oxygen, carbon dioxide and water) might be detrimental to electrical properties but favourable to the thermal conductivity ${ }^{65}$ as they might eliminate the oxygen vacancies at grain boundaries. The slow activation process and the low concentration of these air species acting through the $\mathrm{ZnO}$ films is the defining mechanism in this investigation. The effect of introducing large concentration of water molecules in the sputtering deposition process ${ }^{72}$ and, during the post-deposition annealing treatment ${ }^{73}$ on the electrical properties of $\mathrm{ZnO}$ films has been investigated before. Invariably the presence of water molecules in larger concentrations than the current investigation lead to decrease in mobility due to increase in oxygen related sub-band defect states $^{73}$ that shift the position of the absorption edge to lower energies. The adverse effect of water in the electrical properties of $\mathrm{ZnO}$ films and $\mathrm{ZnO}$ based TFTs has been reported many times. However, small quantities of water have also been reported to result in enhancement of these properties ${ }^{74}$ albeit in films prepared by chemical solution deposition. In the last study it was found that incorporating water molecules, either by water vapour annealing or as an additive in the $\mathrm{ZnO}$ precursor, resulted in higher TFT mobility and lower threshold voltage shift. However, similarly to other reports excessive amounts of water produced acceptor-like traps in the $\mathrm{ZnO}$ film that degraded TFT performance.

Carbon contamination due to environmental factors is expected to be present in our films. If we accept that carbon is conducive to the defect elimination during aging carbon-mediated photolysis might be the operational mechanism. Gurwitz and co-workers demonstrated that carbon-mediated photolysis of CVD-grown $\mathrm{ZnO}$ nanofibres under white light illumination ${ }^{64}$ can lead to oxygen vacancy generation in the surface of the nanofibers. In air, equilibrium was eventually reached between carbon-mediated oxygen depletion and oxygen replacement. Photolysis modified only the surface properties due to the high crystalline quality of the nanofibers ${ }^{75}$. The current films were grown by rf sputtering and the structure is more porous with more intra-band defects spread through the thickness of the films that can absorb visible light photons. Hence the carbon-mediated photolysis could occur throughout the 
thickness of the films resulting in elimination of defects by desorption of mainly loosely bound oxygen. Replenishing of oxygen or absorption of alternative environmental species through mainly grain boundaries cannot be ruled out ${ }^{76}$.

The complex nature of the interplay of donor and acceptor defects states, present at the grain boundaries, and the differing steady state equilibrium achieved under various processing conditions, has been recently demonstrated in the enhancement of Hall mobility of aluminium doped zinc oxide thin films prepared by spray pyrolysis and UV annealed in a nitrogen atmosphere ${ }^{62}$.

Furthermore we should also acknowledge room temperature annealing as another mechanism that might be operational, in addition to the previous mechanisms, during the aging of the films. The effect of room temperature annealing on physical properties of films has been reported before for $\operatorname{short}^{77}$ and extensive periods of time ${ }^{78,79}$. Random motion of atoms taking place at room temperature assisted by various types of defects and local strain over long time might lead to re-constructing of the film, with concomitant elimination of defects especially at grain boundaries that present more degrees of freedom, which makes the film more amenable to laser annealing than the original matrix.

Irrespective of the operational mechanism, aging has a real effect on the electrical properties of the aged films. However, the degree of modification of the electrical properties by laser annealing depends mostly on the properties of the non-processed film which in turn depend largely on the sputtering conditions. Using a high number of pulses is time consuming and not a commercially viable process. However multi-pulse laser annealing is a progressively transformative process while the working thickness of the semiconducting layer in a TFT structure is a lot smaller that the thickness of the films in this investigation. Hence it is postulated that the required number of pulses will be considerably reduced. However, the current report aims to be more qualitative than quantitative and the aim of the paper is to discuss the responsible mechanism for such an improvement in Hall mobility for intrinsic $\mathrm{ZnO}$ while the thickness of the thin films is quite large. Unravelling the mechanism, a new method requiring considerably shorter duration might be devised. The final process might not necessarily require environmental aging but deposition in the proper gas mixture and some short post-processing or accelerated aging under a high pressure gas mixture. Such films can be 
utilised in TFT applications where the lateral movement of carriers in the active layer is affected by the grain boundary defect states ${ }^{80}$ along the columnar grains that zinc oxide is known to form in physical vapour deposition methods.

\section{Conclusions}

We have demonstrated enhancement of Hall mobility in $\mathrm{ZnO}$ thin films deposited by rf magnetron sputtering at ambient temperature $\left(\mathrm{T}<70{ }^{\circ} \mathrm{C}\right)$, via post-deposition excimer laser processing. We specifically demonstrated that enhanced Hall mobility $\left(8.6 \mathrm{~cm}^{2} \mathrm{~V}^{-1} \mathrm{~s}^{-1}\right.$ and $\left.17.3 \mathrm{~cm}^{2} \mathrm{~V}^{-1} \mathrm{~s}^{-1}\right)$ at relatively low carrier concentration $\left(5.6 \times 10^{18} \mathrm{~cm}^{-3}\right.$ and $\left.2.3 \times 10^{18} \mathrm{~cm}^{-3}\right)$ can be achieved with laser processing using low fluence $\left.(52 \mathrm{~mJ} \mathrm{~cm})^{-2}\right)$ immediately after deposition or medium fluence $\left(83 \mathrm{~mJ} \mathrm{~cm}^{-2}\right)$ on aged samples, respectively. The as-deposit sample had a mobility of $1.5 \mathrm{~cm}^{2} \mathrm{~V}^{-1} \mathrm{~s}^{-1}$ and a carrier concentration of $1.3 \times 10^{18} \mathrm{~cm}^{-3}$ while the aged sampled was very resistive $(\mathrm{R}>1 \mathrm{G} \Omega)$. Such films can potentially be used in TFT applications.

\section{Acknowledgements}

HRTEM was performed by Dr Michael Ward under LENNF funded by EPSRC Grant EP/F056311/1 and the University of Leeds. TEM was performed by Dr Simon Newcomb at Glebe Laboratories, Ireland. The authors also would like to thank Shaojun Qi of Birmingham University for the nanoindentation measurements, Dr Naoko Sano of Newcastle University for the etching rate based XPS measurements and Dr Andre Anders of Lawrence Berkeley National Laboratory for many helpful discussions. 


\section{References}

1. Vai, A. T. et al. The transition to the metallic state in polycrystalline n-type doped $\mathrm{ZnO}$ thin films. Zeitschrift fur Anorg. und Allg. Chemie 640, 1054-1062 (2014).

2. Ö̈zür, Ü. et al. A comprehensive review of $\mathrm{ZnO}$ materials and devices. J. Appl. Phys. 98, 1103 (2005).

3. Nakata, M. et al. Effects of thermal annealing on $\mathrm{ZnO}$ thin-film transistor characteristics and the application of excimer laser annealing in plastic-based $\mathrm{ZnO}$ thin-film transistors. Jpn. J. Appl. Phys. 48, 0816081-0816087 (2009).

4. Yang, Y., Yang, S. S. \& Chou, K. Laser-irradiated zinc oxide thin-film transistors fabricated by solution processing. J. Soc. Inf. Disp. 18, 745-748 (2010).

5. Lai, S. C. et al. Extended-pulse excimer laser annealing of $\mathrm{Pb}(\mathrm{Zr} 1-\mathrm{xTix}) \mathrm{O} 3$ thin film on LaNiO3 electrode. J. Appl. Phys. 96, 2779 (2004).

6. Kim, K., Kim, S. \& Lee, S. Y. Effect of excimer laser annealing on the properties of ZnO thin film prepared by sol-gel method. Curr. Appl. Phys. 12, 585-588 (2012).

7. Kim, J. J. et al. Characteristics of laser-annealed $\mathrm{ZnO}$ thin film transistors. Thin Solid Films 518, 3022-3025 (2010).

8. Lee, S. Y., Shim, E. S., Kang, H. S., Pang, S. S. \& Kang, J. S. Fabrication of ZnO thin film diode using laser annealing. Thin Solid Films 473, 31-34 (2005).

9. Yang, Y.-H., Yang, S. S. \& Chou, K.-S. Performance improvements of IGZO and ZnO thinfilm transistors by laser-irradiation treatment. J. Soc. Inf. Disp. 19, 247 (2011).

10. Tsakonas, C. et al. Intrinsic photoluminescence from low temperature deposited zinc oxide thin films as a function of laser and thermal annealing. J. Phys. D. Appl. Phys. 46, (2013).

11. Tsakonas, C. et al. Transparent and Flexible Thin Film Electroluminescent Devices Using HiTUS Deposition and Laser Processing Fabrication. IEEE J. Electron Devices Soc. 4, (2016).

12. Newcomb, S. B. Modern approaches to the preparation of TEM samples. in CONFERENCE SERIES-INSTITUTE OF PHYSICS 179, 357-362 (Philadelphia; Institute of Physics; 1999, 2004). 
13. Sarkar, J. Sputtering materials for VLSI and thin film devices. (William Andrew, 2010).

14. Ellmer, K. \& Mientus, R. Carrier transport in polycrystalline ITO and ZnO:Al II: The influence of grain barriers and boundaries. Thin Solid Films 516, 5829-5835 (2008).

15. Furuta, M. et al. Thermal Stability and Sheet Resistance of Undoped ZnO Films Deposited on Insulators. Electrochem. Solid-State Lett. 12, K74 (2009).

16. Hamad, O., Braunstein, G., Patil, H. \& Dhere, N. Effect of thermal treatment in oxygen, nitrogen, and air atmospheres on the electrical transport properties of zinc oxide thin films. Thin Solid Films 489, 303-309 (2005).

17. Moustaghfir, A. et al. Structural and optical studies of $\mathrm{ZnO}$ thin films deposited by rf magnetron sputtering: influence of annealing. Surf. Coatings Technol. 174, 193-196 (2003).

18. Prins, M. W. J., Grosse-Holz, K.-O., Cillessen, J. F. M. \& Feiner, L. F. Grain-boundarylimited transport in semiconducting $\mathrm{SnO} 2$ thin films: Model and experiments. J. Appl. Phys. 83, 888 (1998).

19. Roth, A. P. \& Williams, D. F. Properties of zinc oxide films prepared by the oxidation of diethyl zinc. J. Appl. Phys. 52, 6685-6692 (1981).

20. Schroder, D. K. Semiconductor material and device characterization. (John Wiley \& Sons, 2006).

21. Blood, P. \& Orton, J. W. The electrical characterization of semiconductors: majority carriers and electron states. 2, (Academic Pr, 1992).

22. Orton, J. W. et al. The Hall effect in polycrystalline and powdered semiconductors. Reports Prog. Phys. 43, 1263-1307 (1980).

23. Rembert, T., Battaglia, C., Anders, A. \& Javey, A. Room Temperature Oxide Deposition Approach to Fully Transparent, All-Oxide Thin-Film Transistors. Adv. Mater. 27, 6090-6095 (2015).

24. Fortunato, E. M. C. et al. Wide-bandgap high-mobility $\mathrm{ZnO}$ thin-film transistors produced at room temperature. Appl. Phys. Lett. 85, 2541-2543 (2004).

25. Seto, J. Y. W. The electrical properties of polycrystalline silicon films. J. Appl. Phys. 46, 5247-5254 (1975). 
26. Lorenz, M. et al. Optical and electrical properties of epitaxial (Mg,Cd)xZn1-xO, $\mathrm{ZnO}$, and $\mathrm{ZnO}:(\mathrm{Ga}, \mathrm{Al})$ thin films on c-plane sapphire grown by pulsed laser deposition. Solid. State. Electron. 47, 2205-2209 (2003).

27. Thompson, C. V. Structure evolution during processing of polycrystalline films. Annu. Rev. Mater. Sci. 30, 159-190 (2000).

28. Bäuerle, D. W. Laser processing and chemistry. (Springer Science \& Business Media, 2013).

29. Priester, L. Grain boundaries: from theory to engineering. 172, (Springer Science \& Business Media, 2012).

30. Keblinski, P., Phillpot, S. R., Wolf, D. \& Gleiter, H. Amorphous structure of grain boundaries and grain junctions in nanocrystalline silicon by molecular-dynamics simulation. Acta Mater. 45, 987-998 (1997).

31. Wang, H. \& Chiang, Y.-M. Thermodynamic Stability of Intergranular Amorphous Films in Bismuth-Doped Zinc Oxide. J. Am. Ceram. Soc. 81, 89-96 (1998).

32. Keblinski, P., Phillpot, S., Wolf, D. \& Gleiter, H. Thermodynamic Criterion for the Stability of Amorphous Intergranular Films in Covalent Materials. Phys. Rev. Lett. 77, 2965-2968 (1996).

33. Masumura, R. A. Enhanced Diffusion Near Amorphous Grain Boundaries in Nanocrystalline and. Energy 1, 31-38 (2000).

34. Dargatz, B. et al. FAST/SPS sintering of nanocrystalline zinc oxide-Part I: Enhanced densification and formation of hydrogen-related defects in presence of adsorbed water. J. Eur. Ceram. Soc. 36, 1207-1220 (2016).

35. Sheetz, R. M., Ponomareva, I., Richter, E., Andriotis, A. N. \& Menon, M. Defect-induced optical absorption in the visible range in $\mathrm{ZnO}$ nanowires. Phys. Rev. B - Condens. Matter Mater. Phys. 80, 1-4 (2009).

36. Srikant, V. \& Clarke, D. R. Optical absorption edge of $\mathrm{ZnO}$ thin films: The effect of substrate. J. Appl. Phys. 81, 6357 (1997).

37. Chattopadhyay, S. et al. Optical property modification of $\mathrm{ZnO}$ : Effect of $1.2 \mathrm{MeV} \mathrm{Ar}$ irradiation. Phys. Status Solidi Curr. Top. Solid State Phys. 8, 512-515 (2011).

38. Kumar, S., Sharma, P. \& Sharma, V. Redshift in Absorption Edge of Cd 1-x Co x S 
Nanofilms. Ieee Trans. Nanotechnol. 13, 343-348 (2014).

39. O'Leary, S. K., Johnson, S. R. \& Lim, P. K. The relationship between the distribution of electronic states and the optical absorption spectrum of an amorphous semiconductor: An empirical analysis. J. Appl. Phys. 82, 3334 (1997).

40. Ellmer, K. Magnetron sputtering of transparent conductive zinc oxide: relation between the sputtering parameters and the electronic properties. J. Phys. D. Appl. Phys. 33, R17-R32 (2000).

41. El Hamali, S. O. et al. Enhanced electrical and optical properties of room temperature deposited Aluminium doped Zinc Oxide (AZO) thin films by excimer laser annealing. Opt. Lasers Eng. 80, 45-51 (2016).

42. Pern, F. J., To, B., Dehart, C., Li, X. \& Glick, S. H. Degradation of ZnO Window Layer for CIGS by Damp-Heat Exposure Preprint. 2008 SPIE PV Reliab. Symp. (2008).

43. Yaklin, M. a, Schneider, D. a, Norman, K., Granata, J. E. \& Staiger, C. L. Impacts of Humidity and Temperature on the Performance of Transparent Conducting Zinc Oxide. IEEE Photovolt. Spec. Conf., 35th 002493-002496 (2010). doi:10.1109/PVSC.2010.5614716

44. Steinhauser, J. et al. Humid environment stability of low pressure chemical vapor deposited boron doped zinc oxide used as transparent electrodes in thin film silicon solar cells. Thin Solid Films 520, 558-562 (2011).

45. Theelen, M. et al. Physical and chemical degradation behavior of sputtered aluminum doped zinc oxide layers for $\mathrm{Cu}(\mathrm{In}, \mathrm{Ga}) \mathrm{Se} 2$ solar cells. Thin Solid Films 550, 530-540 (2014).

46. H??pkes, J. et al. Damp heat stable doped zinc oxide films. Thin Solid Films 555, 48-52 (2014).

47. Rai, R. C. Analysis of the Urbach tails in absorption spectra of undoped $\mathrm{ZnO}$ thin films. $J$. Appl. Phys. 113, (2013).

48. Schönau, S., Ruske, F., Neubert, S. \& Rech, B. Analysis of Urbach-like absorption tails in thermally treated ZnO: Al thin films. Appl. Phys. Lett. 103, 192108 (2013).

49. Long-, O. T. T. P. I. et al. Transport Physics and Device Modeling of Zinc Transport Physics and Device Modeling of Zinc Oxide Thin-Film Transistors Part I : Long-Channel Devices. 
IEEE T. Electron. Dev. 58, 2610 (2011).

50. Muth, J. F., Kolbas, R. M., Sharma, a. K., Oktyabrsky, S. \& Narayan, J. Excitonic structure and absorption coefficient measurements of $\mathrm{ZnO}$ single crystal epitaxial films deposited by pulsed laser deposition. J. Appl. Phys. 85, 7884 (1999).

51. Ashcroft, N. D. \& Mermin, S. Introduction to Solid State Physics, C. Kittel.

52. Donohue, P. P. \& Todd, M. a. Pulse-extended excimer laser annealing of lead zirconate titanate thin films. Integr. Ferroelectr. 31, 285-296 (2000).

53. Bouderbala, M., Hamzaoui, S., Adnane, M., Sahraoui, T. \& Zerdali, M. Annealing effect on properties of transparent and conducting ZnO thin films. Thin Solid Films 517, 1572-1576 (2009).

54. Lad, R. J., Funkenbusch, P. D. \& Aita, C. R. Postdeposition annealing behavior of rf sputtered ZnO films. J. Vac. Sci. Technol. 17, 808-811 (1980).

55. Puchert, M. K. Postdeposition annealing of radio frequency magnetron sputtered $\mathrm{ZnO}$ films. $J$. Vac. Sci. Technol. A Vacuum, Surfaces, Film. 14, 2220 (1996).

56. Hsieh, P.-T., Chen, Y.-C., Wang, C.-M., Tsai, Y.-Z. \& Hu, C.-C. Structural and photoluminescence characteristics of $\mathrm{ZnO}$ films by room temperature sputtering and rapid thermal annealing process. Appl. Phys. A 84, 345-349 (2006).

57. Khan, E. H., Langford, S. C., Dickinson, J. T. \& Boatner, L. A. The interaction of 193-nm excimer laser irradiation with single-crystal zinc oxide: Positive ion emission. J. Appl. Phys. 111, 63101 (2012).

58. Alvarez-Quintana, J., Martínez, E., Pérez-Tijerina, E., Pérez-García, S. A. \& Rodríguez-Viejo, J. Temperature dependent thermal conductivity of polycrystalline ZnO films. J. Appl. Phys. 107, 71-74 (2010).

59. Xu, Y., Goto, M., Kato, R., Tanaka, Y. \& Kagawa, Y. Thermal conductivity of ZnO thin film produced by reactive sputtering. J. Appl. Phys. 111, 84320 (2012).

60. Xiao-Dong, X., Di, M., Shu-Yi, Z., Ai-Hua, L. \& Wasa, K. Thermal diffusivity of film/substrate structures characterized by transient thermal grating method. Chinese Phys. Lett. 25, $176(2008)$. 
61. Zeng, T. \& Chen, G. Phonon Heat Conduction in Thin Films: Impacts of Thermal Boundary Resistance and Internal Heat Generation. J. Heat Transfer 123, 340 (2001).

62. Vai, A. T., Kuznetsov, V. L., Dilworth, J. R. \& Edwards, P. P. UV-induced improvement in ZnO thin film conductivity: a new in situ approach. J. Mater. Chem. C 2, 9643-9652 (2014).

63. Khafizov, M. et al. Thermal conductivity in nanocrystalline ceria thin films. J. Am. Ceram. Soc. 97, 562-569 (2014).

64. Gurwitz, R., Cohen, R. \& Shalish, I. Interaction of light with the ZnO surface: Photon induced oxygen 'breathing,' oxygen vacancies, persistent photoconductivity, and persistent photovoltage. J. Appl. Phys. 115, 33701 (2014).

65. Olorunyolemi, T. et al. Thermal Conductivity of Zinc Oxide: From Green to Sintered State. J. Am. Ceram. Soc. 85, 1249-1253 (2004).

66. Luckyanova, M. N. et al. Thermal conductivity control by oxygen defect concentration modification in reducible oxides: The case of Pr0.1Ce0.9O2- $\delta$ thin films. Appl. Phys. Lett. 104, 6-9 (2014).

67. Dutta, S. et al. Defect dynamics in annealed $\mathrm{ZnO}$ by positron annihilation spectroscopy. $J$. Appl. Phys. 98, 53513 (2005).

68. Meng, X. et al. Grain Boundary Engineering for Achieving High Thermoelectric Performance in n-Type Skutterudites. Adv. Energy Mater. (2017). doi:10.1002/aenm.201602582

69. Kim, S. Il et al. Thermoelectrics. Dense dislocation arrays embedded in grain boundaries for high-performance bulk thermoelectrics. Science 348, 109-14 (2015).

70. Park, H. W., Chung, K. B. \& Park, J. S. A role of oxygen vacancy on annealed $\mathrm{ZnO}$ film in the hydrogen atmosphere. Curr. Appl. Phys. 12, S164-S167 (2012).

71. Steinhauser, J., Fay, S., Oliveira, N., Vallat-Sauvain, E. \& Ballif, C. Transition between grain boundary and intragrain scattering transport mechanisms in boron-doped zinc oxide thin films. Appl. Phys. Lett. 90, 142107 (2007).

72. Nakada, T., Ohkubo, Y. \& Kunioka, A. Effect of water vapor on the growth of textured znobased films for solar cells by dc-magnetron sputtering. Jpn. J. Appl. Phys. 30, 3344-3348 (1991). 
73. Park, H., Park, J.-S., Lee, J. H. \& Chung, K.-B. Thermal Evolution of Band Edge States in ZnO Film as a Function of Annealing Ambient Atmosphere. Electrochem. Solid-State Lett. 15, H133 (2012).

74. Chang, J., Chang, K. L., Chi, C., Zhang, J. \& Wu, J. Water induced zinc oxide thin film formation and its transistor performance. J. Mater. Chem. C 2, 5397 (2014).

75. Shalish, I., Temkin, H. \& Narayanamurti, V. Size-dependent surface luminescence in ZnO nanowires. Phys. Rev. B - Condens. Matter Mater. Phys. 69, 1-4 (2004).

76. Haneda, H., Sakaguchi, I., Watanabe, A., Ishigaki, T. \& Tanaka, J. Oxygen diffusion in singleand poly-crystalline zinc oxides. J. Electroceramics 4, 41-48 (2000).

77. Wang, H.-J. et al. Room-Temperature Annealing of $1 \mathrm{MeV}$ Electron Irradiated Lattice Matched In 0.53 Ga 0.47 As/InP Multiple Quantum Wells. Chinese Phys. Lett. 32, 56102 (2015).

78. Moscatelli, F. et al. Room temperature annealing effects on leakage current of ion implanted p(+)n 4H-SiC diodes. Silicon Carbide Relat. Mater. 2007, Pts 12 600-603, 1027-1030 (2009).

79. Konkova, T., Mironov, S., Korznikov, A. \& Semiatin, S. L. On the room-temperature annealing of cryogenically rolled copper. Mater. Sci. Eng. A 528, 7432-7443 (2011).

80. Hossain, F. M. et al. Modeling and simulation of polycrystalline $\mathrm{ZnO}$ thin-film transistors. $J$. Appl. Phys. 94, 7768-7777 (2003). 